\title{
Polyethylene Mobile Bearing in Total Knee Arthroplasties \\ Design, advantages and preliminary results
}

\begin{abstract}
PAUL DAN SIRBU ${ }^{1}$, VLAD VERINGA ${ }^{2 *}$, OANA VIOLA BADULESCU ${ }^{2 *}$, STEFANITA ARCANA, NORIN FORNA ${ }^{1}$, ANDREI SCRIPCARU ${ }^{1}$, MIHNEA THEODOR SIRBU ${ }^{1}$, OVIDIU ALEXA ${ }^{1}$

${ }^{1}$ Grigore T. Popa University of Medicine and Pharmacy, Faculty of Medicine, Department of Orthopedics and Traumatology, Surgical Sciences (II), 16 Universitatii Str., 700115, lasi, Romania

${ }^{2}$ Grigore T. Popa University of Medicine and Pharmacy, Faculty of Medicine, Department of Pathophysiology, MorfoFunctional Sciences (II), 16 Universitatii Str., 700115, Iasi, Romania

The purpose of this experimental study is to present the design and biomechanical characteristics of mobile bearing postero-stabilised total knee prosthesis type $M C 2^{\circledR}-C^{2} F^{\oplus}$ and to evaluate their use in severe primary knee osteoarthritis. The experimental part was carried out on 20 patients who benefited from mobile bearing postero-stabilised total knee prosthesis type $M C 2^{\circledR}$ in University Hospital Sf Spiridon lasi, Romania. From the radiological point of view there were no mechanical complications in a 3 year follow-up. The functional recovery had a good outcome and patients currently have an active flexion between $70^{\circ}$ and $110^{\circ}$, with extension between $0^{\circ}$ and $-15^{\circ}$. No pain was registered in full weight bearing and in active joint movement. The clinical and radiological outcome at 3 year follow-up is satisfactory and the results convinced the authors that this type of prosthesis is a good option in any primary knee osteoarthritis with no significant bone loss.
\end{abstract}

Keywords: mobile bearing polyethilene, primary knee osteoarthritis, total knee arthroplasty, knee prostheses

Statistics all over the world shows over 100 million people that suffers from osteoarthritis $(O A)$ of the knee and that puts it on the top of the most common causes of disability $[1,2]$.

More than $50 \%$ of the population around the world ( $>65$ years) show $\mathrm{X}$-ray evidence of $\mathrm{OA}$ in one of the joints, thus demonstrating the high incidence of this disease. Furthermore injury-induced osteoarthritis may occur in younger pacients. While OA is equally present in men and women, it appears to be more common among younger men $(<45$ years) and in the older women ( $>45$ years) [35].

In early stages of knee osteoarthritis there are some non-invasive and minimal invasive treatment options such as hyaluronic acid and chondroitin sulfate viscosupplementation with good functional results, pain release and an increased quality of life [6].

Total knee arthroplasty is a reliable procedure with a high implant survival (about 95\%) after 10 years [7-9].

Surgical technique has a highly important role in regard to short and long-term functional results because it generates a balance between ligament forces and the correct alignment of the components of endoprosthesis which, together, grant the stability and the adequate range of motion of the joint.

The endoprostheses component design also plays a considerable role in guaranteeing the proper functioning of the joint and in assuring the least amount of polyethilene $(P E)$ and joint surfaces wear.

It is well known that PE wear is directly proportional with its thickness and with the mechanical stress and strain to which it is exposed to, which should not exceed 10MPa/ $\mathrm{mm} 2$. [10] Moreover, it is crucial that the mechanical stress is distributed as uniformly as possible, rather than as point contact or linear contact (fig 2 C,D).

Lately, mobile-bearing total knee arthroplasty implants are more and more prefered as an alternative to fixedbearing implants because of their advantages related to $\mathrm{PE}$ wear and range of motion.
The balance between the femoral component and the $\mathrm{PE}$ is dependent on their congruence and on the transfer of forces between them, which influences the kinematics. The mobile-bearing prostheses deals with the previously mentioned issues through the axial rotation and anteroposterior movements of the PE on the tibial plateau. This allows for the dispersal of the forces of the femoral condyles that bear on the polyethylene throughout the entire range of motion of the knee, which allows for less wear on the endoprosthesis.

The purpose of this study is to point out the advantages of a mobile bearing total knee prosthesis.

\section{Experimental part}

The experimental part was carried out on 20 patients who benefited from mobile bearing postero-stabilised total knee prosthesis type MC $^{\circledR}$ in University Hospital Sf Spiridon lasi, Romania.

\section{Prosthetic design}

Knee prosthesis type $M C 2^{\circledR}$ is a tricompartimental, postero-stabilized, mobile bearing knee prosthesis. This type of prostheses are used in order to minimize the PE wear, to minimize the stress at all interfaces, to optimize knee function by replicating the natural femoral rollback $(11 \mathrm{~mm})$, to gain the control of the posterior drawer, provide better leverage and to guarante a high flexion capability (fig. 1 A-E)

\section{Femoral component}

The femoral component is made of cobalt-chromium alloy and comes in five right and left sizes available in cemented version and cementless version. It is coated with a double layer of plasma sprayed high porosity titanium and hydroxyapatite, witch provides immediate initial stability and promotes rapid osseointegration (fig. $1 \mathrm{E}^{1}$ ).

The open top of the femoral box allows retrograde intramedullary nailing in case of supracondylar fracture,

\footnotetext{
* email: vlad.veringa@yahoo.com; violabadulescu@yahoo.com; arcana.stefan@gmail.com
} 
although a good option in this kind of fracture may be minimally invasive plate osteosynthesis with systems with angular stability. [11] Femoral box has been optimized to minimize femoral notch resection (fig. 1D). The ribs designed in the lateral condyles decrease weight of the component by $30 \%$ (fig. 1B).

\section{Tibial Tray}

The tibial tray is also made of Cobalt-Chromium alloy, a material whose behaviour in friction is known. The characteristics of friction are optimised by the rectification of the plate and keel, and a high level of polishing. Fig. $\mathrm{IE}^{3}$ It comes in five different sizes, cemented and cementless. It is also coated with a double layer of plasma sprayed high porosity titanium and hydroxyapatite with the same characteristics. The anatomically designed tibial tray fits both right and left knees. Hollow conical core of the keel is designed to acommodate the stem of the mobile bearing.

Mobile bearing $\mathrm{PE}$

The mobile bearing UHMWPE polyethilene vacuumpackaged is also in five sizes available in four thicknesses $(9,11,13,15 \mathrm{~mm})$. The stem has upper cylindrical section and a lower conical section for a total height of $24,5 \mathrm{~mm}$ and it has a broad anterior cut-out which protects it from impingement upon the patellar tendon or the apex of the patella. The dimensions of the mobile bearing PE on the tibial tray allow more than $20^{\circ}$ of rotation while avoiding soft tissue impingement in size to size configurations (fig. $1 \mathrm{E}^{21}$. Mismatch configurations are allowed for the tibial tray and the femoral component. How ever the mobile bearing size must always match the femoral component size. The mobile plate has 2 lateral surfaces that are perfectly congruent with femur between $-5^{\circ}$ and $60^{\circ}$ of flexion (fig. $1 A, C)$.

Kinematics and motion in flexion of $\mathrm{MC}^{2 \otimes}$ prosthesis

One of the most important rules in total knee artrhoplasties is to restore the kinematics of the knee. In order to do this, some parameters must be respected: restoration of the mechanical axis, restoration of the initial joint line, adequate soft tissue balance)

The combined action of anterior cruciate ligament $(A C L)$ and posterior cruciate ligament (PCL) results in posterior translation of the femur in flexion, in a healthy knee. Fig $2 \mathrm{~A}$

When the femur is translated anteriorly in flexion (posterior drawer), soft tissues combined with impingement of the tibial component upon the condyles causes stiffness in flexion and wear of the posterior aspect of PE.

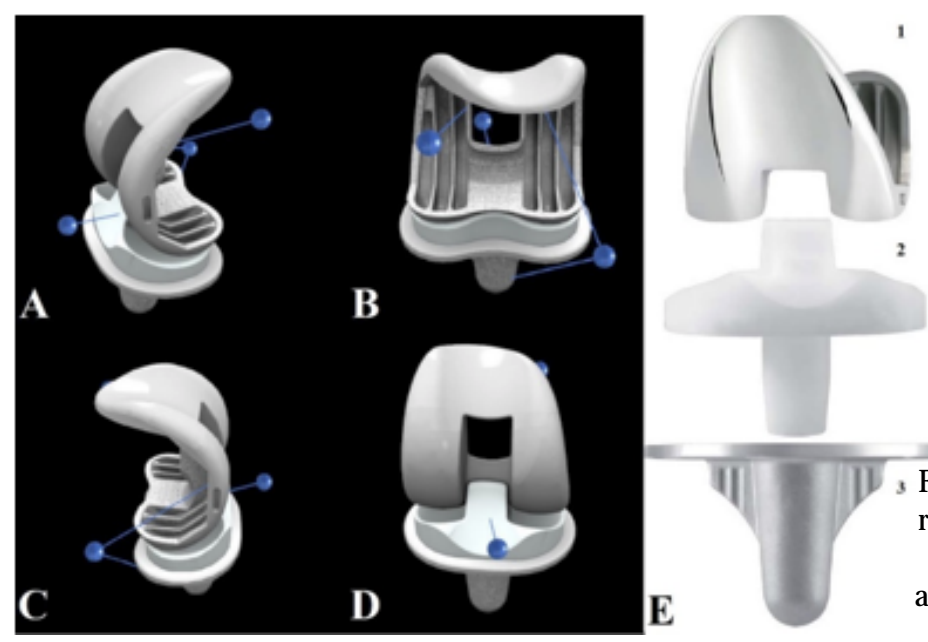

Fig. 1. A-E. MC ${ }^{2 \circledast}$ prosthetic design. Adapted from [10]
This paradoxal kinematics is also detrimental to function because it decreases the extension force of the quadriceps by producing a relative backward shift of the tibial tubercle which decreases the moment arm. Rollback of the PE results in anterior translation of the tibia which increases the extension force of the quadriceps. Fig. 2E,F.

Furthermore the design of the posterior condyles is also influential in achieving high flexion. Thicker posterior condyle provides increased flexion (11mm in $\mathrm{MC}^{2 \mathrm{~B}}$ prostheses versus $8 \mathrm{~mm}$ in most designs) (fig. $2 \mathrm{G}$ ).

The difficulty in designing a total knee prosthesis is to respect all of the following: congruency, level of stresses, kinematics while allowing hyperflexion. This type of prosthesis provide extensive contact area both in extension $\left(1100 \mathrm{~mm}^{2}\right.$ from $-5^{\circ}$ to $\left.60^{\circ}\right)$ and flexion $\left(450 \mathrm{~mm}^{2}\right.$ at $\left.90^{\circ}\right)$ (fig. 2D). Rollback is approximatively $11 \mathrm{~mm}$. Fig. 2B. which induces posterior translation of the femur during flexion. In long-limbed patients high flexion exceeding $150^{\circ}$ is commonly achieved.

Surgical technique

The most important thing at the surgical technique is to guarantee the alignment of the knee and to obtain a balanced ligament stress. The proximal tibial section and the distal femoral sections are orthogonal with respect to the mechanical axis. The ligament stress is evaluated by using braces and block systems which are helping to guide
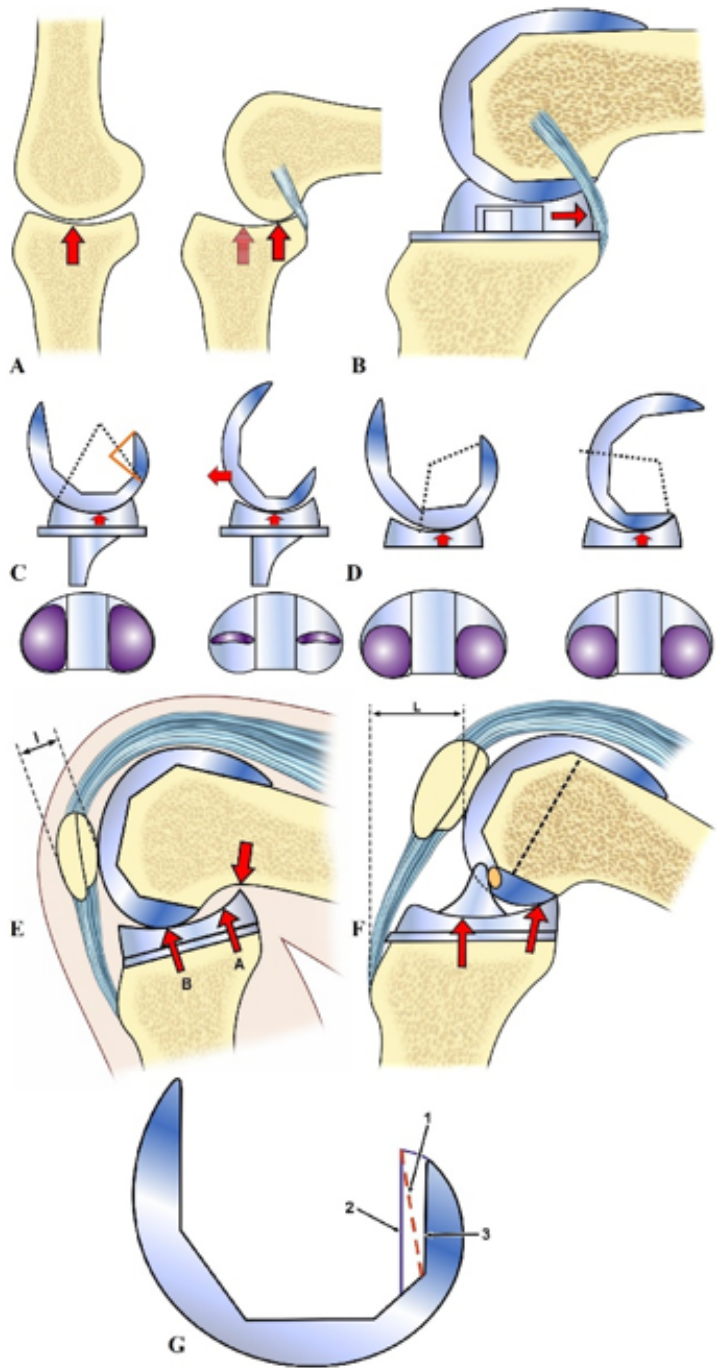

Fig. 2. A-G. Kinematics of $M C^{2 \circledast}$ prosthesis. A. Influence of PCL on rollback in a healthy knee. $\mathbf{B}$. Rollback in a mobile bearing knee. C. Loss of congruency in flexion. $\mathbf{D}$. Congruency both in flexion and extension. E. Posterior drawer in flexion. F. Normal extension mechanism due to rollback of PE. G. Increased thickness of the posterior condyle. Adapted from [10] 
the moves for the ligament release and obtain a rectangular extension gap.

After the joint is exposed, the synovial membrane and osteophytes are removed and a minimum ligament release is done. The femur is drilled in its centre and the centromedullar stem is inserted. The angular cursor now it's adjusted as measured on the preoperative planning and the transverse distal femoral section is made.

The transverse tibial section can be done using centromedullar system or the extra-medullar system. After the two transverse sections are made, the knee gap is measured in full extension and in $90^{\circ}$ flexion with the moveable block unit by $9 \mathrm{~mm}$ to test the knee stability and axis. The femoral anterior, posterior and oblique sections are made using the femoral section blocks. The test components are inserted and the knee stability and axis is verified again in all plans. It is possible to implant any tibial base size since the correspondence is obligatory just betweend the femoral component and the mobile plate, not between the mobile plate and the tibial base. The final component are inserted in place and again the knee stability and axis are verified.

The intraoperative haemostasis was excellent and in the following days there were no important bleeding events. We also used a haemostatic adjuvant, such as tranexamic acid as an antifibrinolytic agent, to assure an excellent haemostasis [12].

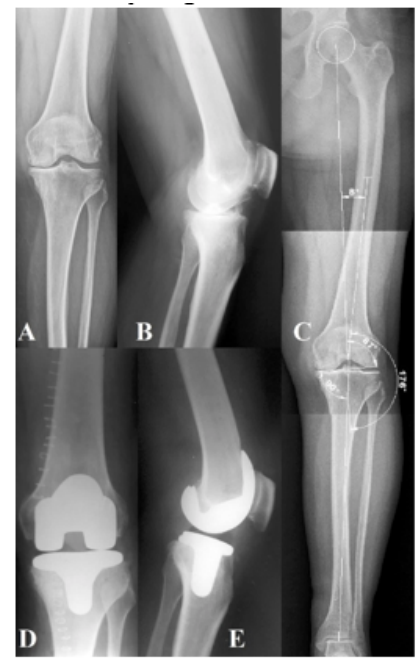

Fig. 3. B.M., F, 59 y.o., Mobile bearing uncemented total knee artrhroplasty. A-E -preoperative and postoperative X-. Rays. A-B -Preoperative X-Rays, $C$-Left lower limb teleradiography with preoperative planning; D-E Postoperative X-Rays. MC ${ }^{2}$ $\mathrm{C}^{2} \mathrm{~F}^{\circledast}$ prosthesis

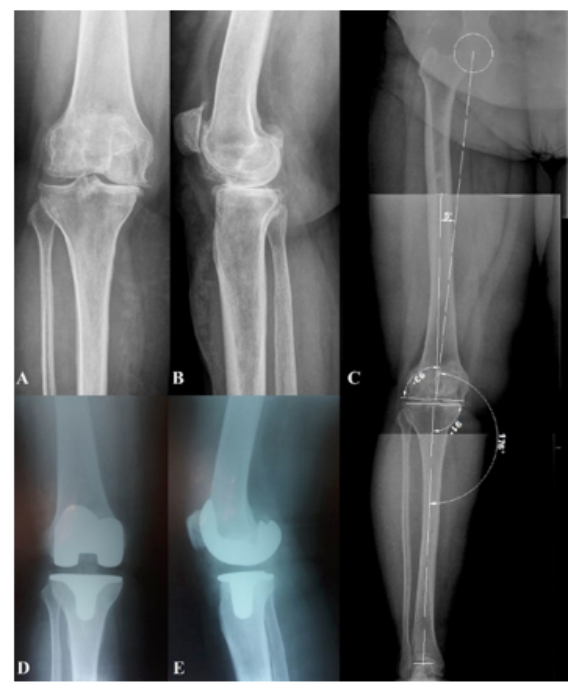

Fig. 4. F. A. F, 58 y.0., Mobile bearing uncemented total knee artrhroplasty. A-E - preoperative and postoperative X-Rays. A-B Preoperative X-Rays, $C$ - Right lower limb teleradiography with preoperative planning; D-E - Postoperative X-Rays. MC ${ }^{2 \circledast}-C^{2} F^{\circledR}$ prosthesis.

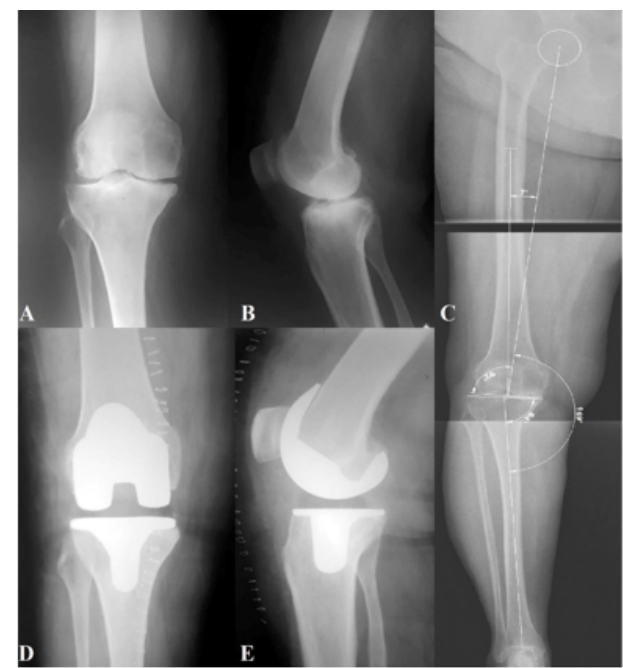

Fig. 5. A. M., F, 70 y.o. Mobile bearing uncemented total knee artrhroplasty. A-E -preoperative and postoperative X-Rays. A-B Preoperative $X$-Rays, $C$-Right lower limb teleradiography with preoperative planning; D-E -Postoperative X-Rays. $\mathrm{MC}^{2 \circledast}-\mathrm{C}^{2} \mathrm{~F}^{\circledast}$ prosthesis

\section{Results and discussions}

We used a tricompartimental, postero-stabilized mobilebearing total knee prosthesis type $\mathrm{MC}^{2 \circledast}-\mathrm{C}^{2} \mathrm{~F}^{\oplus}$ on patients who underwent total knee arthroplasty in our department (Fig. 3-5) with good clinical outcomes, with pain reduced considerably, excellent improvement of joint mobility and life quality.

Immediately after surgery, all patients began walking with total weight bearing with ambulation frame, functional rehabilitation treatmentwith passive motion on arthromotor and assisted active progressive exercises.

Although skin necrosis around the incision and little soft tissue damage is often seen in this kind of surgery, we did not have any complications, no soft tissue damage and no negative preasure therapy was used [13].

All patients were regularly monitored after the surgery, both clinically and imagistic, at 3 weeks, 6 weeks, 3 months and every 3 months after, including rehabilitation program.

From the radiological point of view there were no mechanical complications in a 3 year follow-up.

The functional recovery has a good outcome and patients currently have an active flexion between $70^{\circ}$ and $110^{\circ}$, with extension between $0^{\circ}$ and $-15^{\circ}$. No pain was registered in full weight bearing and in active joint movement.

Poirier etal. compared in a study mobile-bearing versus fixed-bearing total knee implants and the results were similar, with no significant differences in the clinical outcomes between fixed-bearing and mobile-bearing inserts of the same TKA model. Although the mobile bearing knees had a better radiographic appearance, this did not translate to better clinical outcomes. In practice, the superiority of mobile bearings is solely theoretical [14].

Vogt et al. concluded in a retrospective study on 101 patients with 126 knees treated with a mobile-bearing system that this type of knee prostheses demonstrates a good behavior in case of slight malrotation of the tibial and femoral components and postoperative axis deviations. Axis deviations up to $10^{\circ}$ of valgus or $4^{\circ}$ of varus did not interfere either with clinical or functional results or long term radiographic outcome of the involved prostheses [15].

Pais-Brito et al. evaluated in 2015157 consecutive knees wich underwent total knee arthroplasty, 82 fixed-bearing system and 75 mobile-bearing system. At a 5 year follow- 
up in terms of pain on walking, pain on ascending/ descending stairs, patella-femoral pain, they found a statistically significant advantage for the mobile-bearing group [16].

In 2014 Okamoto et al. studied the weight bearing total knee arthroplasty kinematics in step-up activity; 37 patients with 40 knees were randomized to a MB versus FB system. They found no statistically difference between the two groups in post-operative range of motion, angle and component alignment. Fixed-bearing and mobile-bearing groups demonstrated no major kinematic differences in terms of antero-posterior translation and axial rotation during step-up activity, although a wider range of axial rotation was registered in MB systems [17].

Artz et al. [18] surveyed FB and MB TKAs regarding kneeling ability, which is considered an importantoutcome by approximately $50 \%$ of patients. Including 206 TKAs, selfreported kneeling ability from OKS at 1 and 2 years after surgery was significantly higher in FB (from 4\% preoperative to $28 \%$ at 2 years after surgery) versus MB group (from 3\% preoperative to $11 \%$ at 2 years after surgery), with a significant correlation for Western Ontario and McMaster Universities Arthritis Index (WOMAC) pain and function scores; interestingly, in spite of the difference in reported ability to kneel, ROM and WOMAC score were similar in both groups after surgery.

\section{Conclusions}

The tricompartimental, postero-stabilized mobilebearing total knee prosthesis type $M C^{2 \Theta}-C^{2} F^{\circledast}$ is a viable option in any primary osteoarthritis which needs total knee arthroplasty; with many advantages such as intraoperatory slight malrotation of the tibial component, and axis deviations.

In the same time, mobile-bearing type $\mathrm{MC}^{2}{ }^{2}$ prostheses has the advange to minimize the PE wear, to minimize the stress at all interfaces, to optimize knee function by replicating the natural femoral rollback, to gain the control of the posterior drawer and provide better leverage and to guarante a high flexion capability.

The clinical and radiological outcome at 3 year followup is satisfactory and the results convinced the authors that this type of prosthesis is a good option in any primary knee osteoarthritis with no significant bone loss.

\section{References}

1.HINMAN RS, HUNT MA, CREABY MW, WRIGLEY T, MCMANUS FJ, BENNELL KL. Hip muscle weakness in individuals with medial knee osteoarthritis. Arthritis Care Res.2010;62:1190.3.

2.HUANG CH, LIAU JJ, CHENG CK. Fixed or mobile-bearing total knee arthroplasty. J Orthop Surg Res 2007;2:1.

3.HEINERT G, KENDOFF D, PREISS S, et al. Patellofemoral kinematics in mobile-bearing and fixed-bearing posterior stabilised total knee replacements: a cadaveric study. Knee Surg Sports Traumatol Arthrosc 2011;19:967-72.
4.DENNIS DA, CLAYTON ML, O'DONNELL S, et al., Posterior cruciate condylar total knee arthroplasty. Average 11-year follow-up evaluation, Clin Orthop, 1999;281:168-76.

5.SCHAI PA, THORNHILL TS, SCOTT RD, Total knee arthroplasty with the PFC system: Results at a minimum of ten years and survivorship analysis, J Bone J oint Surg (Br), 1998;80:850-58.

6.SIRBU, P.D., TUDOR, R, BADULESCU, O.V., Hyaluronic Acid and Chondroitin Sulfate Viscosupplementation in Severe Arthropathy of Hemophilic Patients,Rev. Chim. (Bucharest), 68, no. 5, 2017, p. 10281030

7.BANKS S, BELLEMANS J, NOZAKI H, et al., Knee motion during maximum flexion in fixed and mobile-bearing arthroplasties, Clin Orthop, 2003;410:131-38.

8.HINMAN RS, BENNEL K, METCALF B, CROSSELY K. Delayed onset of quadriceps activity and altered knee joint kinematics during stair stepping in individuals with knee osteoarthritis. Arch Phys Med Rehabil. 2002;83:1080-6.

9.RITTER MA, CAMPBELL E, FARIS PM. Long-term survival analysis of the posterior cruciate condylar total knee arthroplasty: a 10-year evaluation. J Arthroplasty 1989; 4:293-6.

10.*** http://c2f-implants.xnov.com/EN/Product-knee.php\#mc2

11.SIRBU PD; CARATA E; PETREUS T; ASAFTEI R; BOTEZ P. Minimally Invasive Plate Osteosynthesis with Systems with Angular Stability in Complex Distal Femoral Fractures. Design, Biomechanics and Clinical Results. AT-EQUAL 2009: 2009 ECSIS SYMPOSIUM ON ADVANCED TECHNOLOGIES FOR ENHANCED QUALITY OF LIFE: LAB-RS AND ARTIPED 2009

12.BADULESCU, O.V., TUDOR, R., FRIEDL, W., BLAJ, M., SIRBU, P,D., Efficiency of Tranexamic Acid in Management of Surgical Orthopedic Bleeding in Patients with Haemophilia,Rev, Chim. (Bucharest), 68, no. 3, 2017, p. 627-630

13.CIUNTU, B.M., VASILUTA, C., NEGRU, R., HULTOANA, R., CIUNTU, R.,GEORGESCU, S.O., SIRBU, P.D., AZOICAI, D., Negative Pressure Therapy in the Surgical Treatment of Diabetic FootRev. Chim. (Bucharest), 68, no. 7, 2017, p. 1648-1651.

14.POIRIER N, GRAF P, DUBRANA F. Mobile-bearing versus fixedbearing total knee implants. Results of a series of 100 randomised cases after 9 years follow-up. Orthop Traumatol Surg Res 2015;101:S18792.

15.VOGT J.C. et al, LCS mobile-bearing total knee replacement. A 10year's follow-up study. Orthopaedics \& Traumatology: Surgery \& Research (2009) 95, 177-182

16.PAIS-BRITO JL, RAFOLS-URQUIZA B, GONZALEZ-MASSIEU L, et al. Reduced patellofemoral and walking pain with mobilebearing vs. fixed-bearing total knee replacements: a midterm prospective analytic study. Acta Orthop Traumatol Turc 2015;49:375-81.

17.OKAMOTO N, NAKAMURA E, NISHIOKA H, et al. In vivo kinematic comparison between mobile-bearing and fixedbearing total knee arthroplasty during step-up activity. J Arthroplasty 2014;29:2393-6.

18.ARTZ NJ, HASSABALLA MA, ROBINSON JR, et al. Patient Reported Kneeling Ability in Fixed and Mobile Bearing Knee Arthroplasty. J Arthroplasty 2015;30:2159-63.

Manuscript received: 26.06 .2019 